\title{
Biosynthesis of the exopolysaccharide galactoglucan in Sinorhizobium meliloti is subject to a complex control by the phosphate- dependent regulator PhoB and the proteins ExpG and MucR
}

\author{
Silvia Rüberg, Alfred Pühler and Anke Becker
}

Lehrstuhl für Genetik, Fakultät für Biologie, Universität Bielefeld, Postfach 100131, D-33501 Bielefeld, Germany

\author{
Author for correspondence: Anke Becker. Tel: +49521 1065607 . Fax: +49521 1065626 \\ e-mail: Anke.Becker@genetik.uni-bielefeld.de
}

\begin{abstract}
The soil bacterium Sinorhizobium meliloti (Rhizobium meliloti) has the ability to produce the alternative exopolysaccharide galactoglucan (EPS II) in addition to succinoglycan (EPS I). In the wild-type strain EPS II production is induced by phosphate-limiting conditions or by extra copies of the exp gene cluster. Based on similarities to transcriptional regulators of the MarR family, an additional putative regulatory gene, expG, was identified in the exp gene cluster. Using exp-lacz transcriptional fusions, a stimulating effect of extra copies of this expG gene on the transcription of all exp complementation groups was determined. Phosphate limitation also resulted in increased expression of the exp-lacz fusions. This increase was reduced in strains characterized by a deletion of expG. The previously reported high level of exp gene transcription in a muCR mutant was further elevated under phosphate-limiting conditions. The $\operatorname{expA,} \operatorname{expD,} \operatorname{expG}$ and $\exp E$ promoters contain sequences with similarities to the PHO box known as the PhoB-binding site in phosphate-regulated promoters in Escherichia coli. The S. meliloti phoB gene was required for the activation of exp gene expression under phosphate limitation, but not for induction of exp expression by MucR or ExpG.
\end{abstract}

Keywords: phosphate regulation, $\mathrm{PHO}$ box, Rhizobium meliloti

\section{INTRODUCTION}

The soil bacterium Sinorhizobium meliloti (Rhizobium meliloti) has the ability to produce the two acidic exopolysaccharides EPS I (succinoglycan) and EPS II (galactoglucan). The EPS I produced under standard conditions is required for nodule invasion of Medicago sativa (Leigh et al., 1985; Battisti et al., 1992; Leigh \& Walker, 1994). It is composed of octasaccharide repeating units containing one galactose and seven glucose residues and can be decorated by acetyl, succinyl and pyruvyl groups (Reinhold et al., 1994). The $36 \mathrm{~kb}$ exo/exs gene cluster directing the biosynthesis of EPS I was extensively studied and functions of the gene products were proposed (Buendia et al., 1991; Becker et al., 1993a, b, c, 1995a, b; Glucksmann et al., 1993a, b; Müller et al., 1993; Reuber \& Walker, 1993).

EPS II can functionally replace the structurally different EPS I in nodule invasion (Glazebrook \& Walker, 1989; Zhan et al., 1989; González et al., 1996). EPS II is composed of alternating glucose and galactose residues which are decorated by acetate and pyruvate (Glazebrook \& Walker, 1989). Recently, we reported on the $32 \mathrm{~kb}$ exp gene cluster directing the biosynthesis of EPS II and on the inferred properties of the encoded gene products (Becker et al., 1997).

Under normal culture conditions, S. meliloti wild-type cells produce large amounts of EPS I and synthesize almost no EPS II. Zhan et al. (1991) showed that under phosphate-limiting conditions the wild-type strain is able to produce the alternative exopolysaccharide EPS II. Moreover, extra copies of the exp gene cluster resulted in the biosynthesis of EPS II (Glazebrook \& Walker, 1989; Zhan et al., 1989). The production of EPS II was also observed in the presence of a mutation in either of the regulatory genes $\operatorname{expR}$ (Glazebrook \& Walker, 1989) or mucR (Keller et al., 1995). In addition, it was shown that all $\exp$ complementation groups are transcribed at a substantially elevated level in a mucR mutant (Keller et al., 1995; Becker et al., 1997). While 
the regulatory genes $\exp R$ and $m u c R$ are located on the chromosome, an additional regulatory gene, $\exp G$, was identified on megaplasmid 2 in the exp gene cluster (Becker et al., 1997). The proposed function of the $\exp G$ gene product as a transcriptional regulator is based on weak homologies between ExpG and the PapX protein of Escherichia coli, a putative regulator of genes directing the biosynthesis of $\mathrm{P}$ pili (Marklund et al., 1992). The strongest homologies were found to a conserved region of 35 amino acids which was described as the MarR family signature (Dehoux \& Cossart, 1995). Transcriptional activators belonging to the MarR family bind DNA through a helix-turn-helix motif (Cohen et al., 1993; Sulavik et al., 1995). Recently, Astete \& Leigh (1996) identified in S. meliloti the gene $m u c S$ which is identical to $\exp G$. mucS is required for EPS II induction by extra copies of the exp gene cluster. This gene is also required for the activation of the expression of one gene in the $\exp E$ operon under phosphate limitation.

In this report, we investigate the complex regulation of exp gene expression by phosphate and the regulatory genes $\exp G$ and $m u c R$. Apart from an influence on the exp gene transcription by each factor alone, we focus on the interaction between the regulatory pathways involving phosphate availability and the regulators ExpG and MucR.

\section{METHODS}

Bacterial strains and plasmids. Strains and plasmids used in this study are listed in Table 1.

Media and growth conditions. E. coli strains were grown in Penassay broth (Difco) or in LB medium (Sambrook et al., 1989 ) at $37^{\circ} \mathrm{C}$. S. meliloti strains were grown in TY (Beringer, 1974) or LB (Sambrook et al., 1989) medium at $30^{\circ} \mathrm{C}$. To study the effect of limiting phosphate on exp gene expression, the $S$. meliloti strains were grown in a MOPS-buffered medium described by Zhan $e t$ al. (1991). For the $\beta$-galactosidase assay, strains grown for $48 \mathrm{~h}$ in LB or TY liquid medium were washed once with MOPS medium containing either $2 \mathrm{mM}$ phosphate (high phosphate) or $0.1 \mathrm{mM}$ phosphate (low phosphate). A volume of washed cells corresponding to $75 \mu \mathrm{l}$ stationary culture was used to inoculate $10 \mathrm{ml}$ of the appropriate MOPS medium.

Antibiotics were supplemented as required at the following concentrations $\left(\mu \mathrm{g} \mathrm{ml}^{-1}\right)$ : streptomycin $(\mathrm{Sm}), 600$; tetracycline (Tc), 10; gentamicin (Gm), 40; neomycin (Nm), 120 for $S$. meliloti and tetracycline, 10; gentamicin, 10; ampicillin (Ap), 100 ; and kanamycin (Km), 50 for E. coli.

DNA biochemistry. Preparation of plasmid DNA, DNA restriction, agarose gel electrophoresis, cloning procedures and transformations of $E$. coli cells were carried out according to established protocols (Sambrook et al., 1989). Southern hybridizations were performed according to Kessler (1992). Total DNA from rhizobia was isolated according to Meade $e t$ al. (1982).

DNA sequencing. DNA sequencing to verify new plasmid constructs or mutations was carried out using the "AutoRead Sequencing kit' (Pharmacia) according to a protocol devised by Zimmermann et al. (1990). Sequence data were obtained and processed using the ALF DNA Express Sequencer (Pharmacia) according to the manufacturer's instructions.
Construction of the S. meliloti expG deletion mutant. Plasmid pARII/ 1 inv $\Delta \exp G$ characterized by a deletion comprising 490 nucleotides of the $3^{\prime}$-terminus of the $\exp G$ coding region and 17 nucleotides downstream of $\exp G$ was transferred by conjugation from the broad-host-range mobilizing strain $E$. coli S17-1 (Simon et al., 1983) to $S$. meliloti 2011 as described by Simon (1984). Homogenotization of the $\exp G$ deletion was carried out as described by Masepohl et al. (1988). Selection by neomycin resistance conferred by plasmid pARII/1inv $\Delta \exp G$ resulted in the identification of a merodiploid strain carrying the plasmid integrated into the genome. This strain was cultured in liquid LB medium without antibiotic stress until stationary phase. Subsequently, homogenotes that lost neomycin resistance were tested for the absence of the vector and the presence of the $\exp G$ deletion by Southern hybridization. The resulting strain was designated $\mathrm{SmSR} \Delta \mathrm{G}$.

Interposon mutagenesis. Using appropriate restriction sites, a promoterless lacZ-Gm interposon (Becker et al., 1995c) was inserted in the direction of transcription into fragments of the exp gene region, which were subcloned into the mobilizable suicide vector pK18mob (Schäfer et al., 1994). Resulting hybrid plasmids were transferred from E. coli S17-1 (Simon et al., 1983) to the $S$. meliloti expG deletion mutant SmSR $\Delta \mathrm{G}$ as described by Simon (1984). Homogenotization of the lacZ$\mathrm{Gm}$ insertions was carried out as described by Masepohl et al. (1988). All homogenotes were verified by Southern hybridization. S. meliloti exp-lacZ/mucR double mutants (Becker et al., 1997), exp-lacZ/mucR/ $\operatorname{expG~triple~mutants,~}$

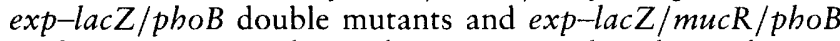
triple mutants were obtained via $\Phi$ M12-mediated transduction (Finan et al., 1984).

$\boldsymbol{\beta}$-Galactosidase assay of $\boldsymbol{S}$. meliloti strains carrying lacZ-Gm insertions. $S$. meliloti strains were grown to an $\mathrm{OD}_{600}$ of $0.6-0.8$ in MOPS medium initially containing $0.1 \mathrm{mM}$ phosphate or $2 \mathrm{mM}$ phosphate. The assay for $\beta$-galactosidase activity was carried out according to the protocol devised by Miller (1972). Relative $\beta$-galactosidase units were calculated per cell number according to Miller (1972).

\section{RESULTS}

\section{Phosphate limitation stimulates the expression of exp-lacZ transcriptional fusions}

Previous complementation and operon analyses using exp-lacZ transcriptional fusions revealed the presence of five transcriptional units in the exp gene cluster (Glazebrook \& Walker, 1989; Becker et al., 1997). To determine whether the transcription of these five exp complementation groups is affected by phosphate limitation, the $\beta$-galactosidase activities of one representative exp-lacZ transcriptional fusion for each exp operon (Becker et al., 1997) were determined in MOPS medium with $0.1 \mathrm{mM}$ phosphate (low phosphate) or $2 \mathrm{mM}$ phosphate (high phosphate) added. The transcription of the exp-lac $Z$ fusions was studied in wildtype and mucR mutant backgrounds.

In the wild-type background exp-lac $Z$ fusions showed only basal levels of $\beta$-galactosidase activity when the strains were cultured in MOPS medium containing $2 \mathrm{mM}$ phosphate (Fig. 1). In MOPS medium containing $0.1 \mathrm{mM}$ phosphate the expression of the expA1-lacZ and $\exp C-l a c Z$ fusions was stimulated about eightfold, whereas the expression of the expE2-lacZ and the 
Table 1. Strains and plasmids

\begin{tabular}{|c|c|c|}
\hline Strain/plasmid/phage & Relevant characteristics & Source/reference \\
\hline \multicolumn{3}{|l|}{ S. meliloti } \\
\hline $\operatorname{Rm} 2011$ & Wild-type, $\mathrm{Nx}^{\mathrm{r}} \mathrm{Sm}^{\mathrm{r}}$ & Casse et al. (1979) \\
\hline $\operatorname{Rn} 3131$ & $\operatorname{Rm} 2011, \operatorname{mucR} 31:: \operatorname{Tn} 5$ & Keller et al. (1995) \\
\hline $\mathrm{RmI01}$ & $\begin{array}{l}\mathrm{Rm} 2011, \mathrm{Spc}^{r} \text { cassette of } \mathrm{pHP} 45 \Omega \text { inserted into the Pmacl site of } \\
\text { mucR (mucR101-Spc) }\end{array}$ & Becker et al. (1997) \\
\hline $\operatorname{RmAR} 1014$ & 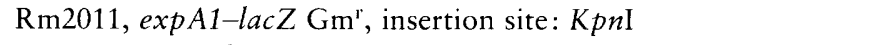 & Becker et al. (1997) \\
\hline RmAR 1016 & 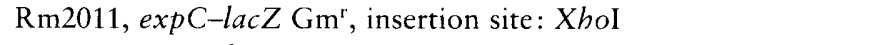 & Becker et al. (1997) \\
\hline RmAR 1018 & $\mathrm{Rm} 2011, \exp G-l a c Z \mathrm{Gm}^{\mathrm{r}}$, insertions site: StuI & Becker et al. (1997) \\
\hline $\operatorname{RmAR} 1020$ & 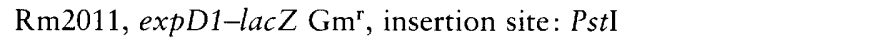 & Becker et al. (1997) \\
\hline $\operatorname{RmAR} 1022$ & 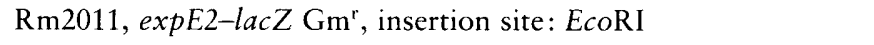 & Becker et al. (1997) \\
\hline RmAR 1114 & $\mathrm{Rm} 3131, \exp A 1-l a c Z \mathrm{Gm}^{r}$, insertion site $: K p n \mathrm{I}$ & Becker et al. (1997) \\
\hline $\operatorname{RmAR} 1116$ & $\mathrm{Rm} 3131, \exp C-$ lacZ Gm $\mathrm{Gm}^{\mathrm{r}}$, insertion site: Xbol & Becker et al. (1997) \\
\hline RmAR 1118 & $\mathrm{Rm} 3131, \exp G-l a c Z \mathrm{Gm}^{\mathrm{r}}$, insertion site: StuI & Becker et al. (1997) \\
\hline RmAR 1120 & 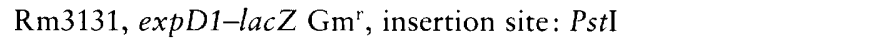 & Becker et al. (1997) \\
\hline $\mathrm{RmAR} 1122$ & 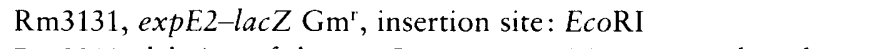 & Becker et al. (1997) \\
\hline $\mathrm{SmSR} \Delta \mathrm{G}$ & $\begin{array}{l}\text { Rm2011, deletion of the } \exp G \text { gene comprising } 490 \text { nucleotides } \\
\text { of the } 3^{\prime} \text {-terminus of the } \exp G \text { coding region and } 17 \text { nucleotides } \\
\text { downstream of } \exp G\end{array}$ & This work \\
\hline $\mathrm{RmH} 615$ & $\mathrm{Lac}^{-}, \Omega p h o B 3:: \operatorname{Tn} V$ & Bardin \& Finan (1998) \\
\hline $\mathrm{RmH} 406$ & sphoB3:: Tn5-132 & $\begin{array}{l}\text { T. M. Finan, McMaster University, } \\
\text { Hamilton, Ontario, Canada }\end{array}$ \\
\hline \multicolumn{3}{|l|}{ E. coli } \\
\hline XL-1 Blue & recA1 lac $\left[\mathrm{F}^{\prime}\right.$ proAB lac $\left.\mathrm{I}^{\mathrm{q}} \mathrm{Z} \Delta \mathrm{M} 15 \mathrm{Tn} 10\left(\operatorname{tet}^{\mathrm{r}}\right)\right]$ thi & Bullock et al. (1987) \\
\hline $\mathrm{S} 17-1$ & $\begin{array}{l}\text { E. coli } 294 \text {, thi RP4-2-Tc::Mu-Km::Tn7 integrated into the } \\
\text { chromosome }\end{array}$ & Simon et al. (1983) \\
\hline \multicolumn{3}{|l|}{ Plasmid } \\
\hline $\mathrm{pARII} / \operatorname{linv} \Delta \exp G$ & $\begin{array}{l}\text { pK } 18 \text { mob containing a } 2.9 \mathrm{~kb} K p n \mathrm{I}-B g l \mathrm{II} \text { fragment with a } \\
\text { deletion comprising } 490 \text { nucleotides of the } 3^{\prime} \text {-terminus of the } \\
\exp G \text { coding region and } 17 \text { nucleotides downstream of } \exp G\end{array}$ & A. Roxlau \\
\hline pPHU231 & pRK290 derivative, broad-host-range vector & Hübner et al. (1991) \\
\hline pSR 1018 & $\begin{array}{l}\text { pPHU231 containing a } 1 \mathrm{~kb} \text { Pst I fragment with the } \exp G \text { gene } \\
\text { under control of its own promoter }\end{array}$ & This work \\
\hline pSR 1018-5 & $\begin{array}{l}\text { pPHU231 containing a } 0.7 \mathrm{~kb} \text { RsaI-Pst I fragment with the } \exp G \\
\text { gene under control of the E. coli lac promoter }\end{array}$ & This work \\
\hline pK18mob & pUC18 derivative, $l a c Z \alpha \mathrm{Km}^{\mathrm{r}}$, mob site & Schäfer et al. (1994) \\
\hline \multicolumn{3}{|l|}{ Phage } \\
\hline$\Phi M 12$ & S. meliloti transducing phage & Finan et al. (1984) \\
\hline
\end{tabular}

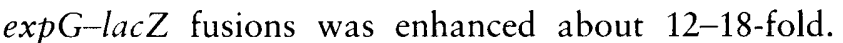

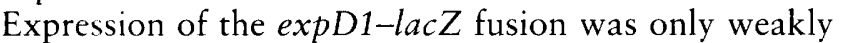
influenced by phosphate starvation. Growth in MOPS medium with $0 \cdot 1 \mathrm{mM}$ phosphate resulted in a twofold increase of $\beta$-galactosidase activity.

In contrast to the $\beta$-galactosidase activities mediated by exp-lacZ fusions in the wild-type background, $\beta$ galactosidase activities of the $m u c R$ mutant carrying the exp-lacZ fusions were already detectable at a substantial level under high phosphate conditions (Fig. 1). Negative regulation of exp gene transcription by $m u c R$ has already been shown for $S$. meliloti strains grown in LB medium (Becker et al., 1997). However, phosphate limitation of the $m u c R$ mutant resulted in an additional increase in the expression of the exp-lacZ fusions (Fig.
1). This indicates that MucR is dispensable for the activation of exp gene expression under phosphatelimiting conditions.

\section{An expG mutation reduces the stimulating effect of phosphate limitation on the expression of the expA and expE operons}

Recently it was reported that under phosphate limitation the regulatory gene $\exp G$ is essential for the activation of the transcription of one gene in the expE operon (Astete \& Leigh, 1996). To investigate the role of the $\exp G$ gene in the phosphate-mediated regulation of additional exp operons, the $\beta$-galactosidase activities of the expA1-lacZ, expD1-lacZ and expE2-lacZ fusions 


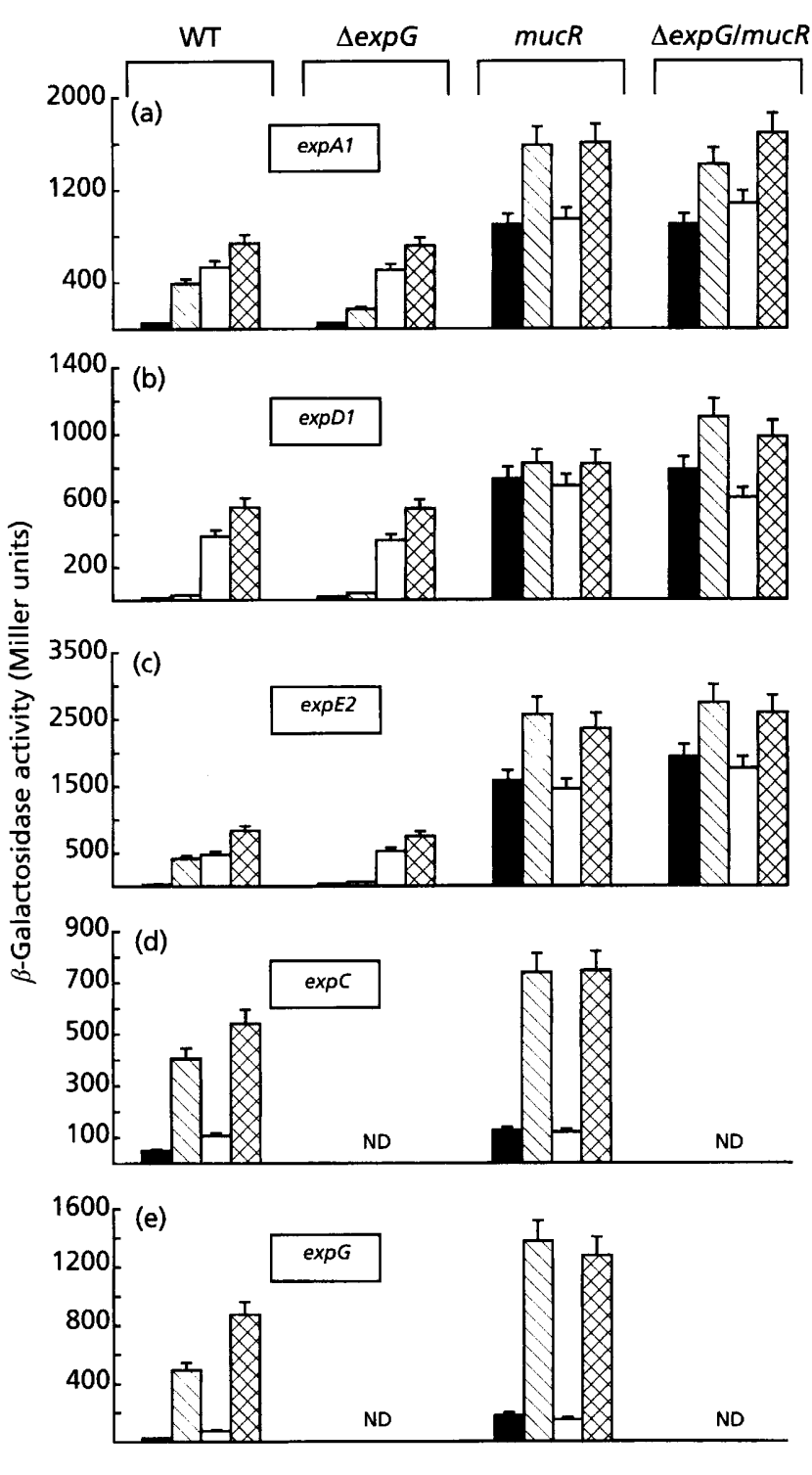

Fig. 1. Histogram showing the $\beta$-galactosidase activities expressed by exp-lac $Z$ transcriptional fusions in the wild-type Rm2011, the expG mutant SmSR $\Delta G$, the mucR mutant Rm3131 and the $\triangle \exp G-m u c R$ double mutant dependent on phosphate concentration and copy number of the expG gene. Relative $\beta$ -

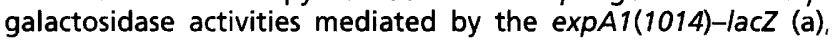
$\operatorname{expD1}(1020)-l a c Z$ (b), expE2(102.2)-lacZ (c), expC(1016)-lacZ (d) and $\exp (1018)-l a c Z$ (e) transcriptional fusions in the background of the wild-type (WT), the expG deletion mutant:



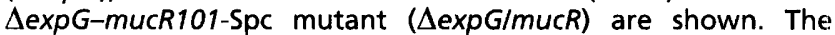
cells were grown in MOPS medium with $2 \mathrm{mM}$ phosphate or $0.1 \mathrm{mM}$ phosphate added in the presence or absence of extra copies of the expG gene on plasmid PSR1018-5 as indicated by the different shading of the bars $(0,2 \mathrm{mM}$ phosphate; $\mathbb{}$, $0.1 \mathrm{mM}$ phosphate; $\square, 2 \mathrm{mM}$ phosphate + pSR1018-5; $0.1 \mathrm{mM}$ phosphate + pSR 1018-5). The $\beta$-galactosidase activity values are means of at least five independent assays. The background $\beta$-galactosidase activity of the wild-type strain Rm2011 was $4 \pm 1$ Miller units. Error bars denote 1 SD. ND, Not determined.

were determined in the $\exp G$ deletion $(\Delta \exp G)$ mutant $\operatorname{SmSR} \Delta \mathrm{G}$. The observed increase in the expression of the $\exp A 1-l a c Z$ and $\operatorname{expE2-lacZ}$ transcriptional fusions under phosphate limitation was reduced in the $\Delta \exp G$ mutant (Fig. 1). In the wild-type strain $\beta$-galactosidase activities mediated by the expA1-lacZ transcriptional fusion were enhanced sevenfold under phosphate limitation. However, phosphate limitation resulted in only a threefold increase in $\beta$-galactosidase activity in the $\Delta \exp G$ background (Fig. 1). The $\exp G$ deletion had a stronger effect on the expression of the expE2-lacZ transcriptional fusion under phosphate limitation. Whereas $\beta$-galactosidase activity in the wild-type strain

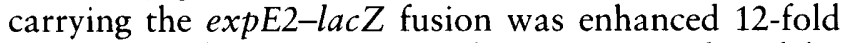
under phosphate starvation, the activity mediated by this fusion in mutant SmSR $\Delta G$ was only enhanced twofold (Fig. 1). No effect of the $\exp G$ deletion could be

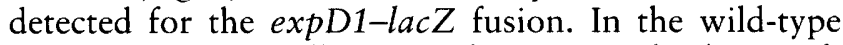
background as well as in the $\Delta \exp G$ background, transcription of the $\operatorname{expD1}$ gene was only increased at a very low level under phosphate limitation. Compared to the wild-type background, the expression of the expD1lacZ fusion was not decreased.

Previously, we have demonstrated that a mutation in the regulatory gene $m u c R$ resulted in the enhanced expression of the five $\exp$ transcriptional units. To analyse whether $\exp G$ influences the effect of the mucR mutation on $\exp$ gene transcription, the $\beta$-galactosidase activities of the expA1-lacZ, expD1-lacZ and expE2-lacZ fusions were measured in a $\Delta \exp G-m u c R$ double mutant. In comparison to the results obtained with the $m u c R$ mutant, no significant difference was detected in the $\Delta \exp G-m u c R$ double mutant under high or under low phosphate concentrations (Fig. 1). This indicates that the deletion of the $\exp G$ gene had no influence on the level of $\exp$ gene expression in a mucR mutant.

\section{Extra copies of the expG gene stimulate the transcription of the five exp operons}

To study the effect of the $\exp G$ gene dosage on $\exp$ gene regulation, the $\exp G$ gene was subcloned under the control of its own promoter and the E. coli lac promoter in the low copy vector pPHU231 (Hübner et al., 1991). The resulting plasmids pSR1018 (native promoter), pSR1018-5 (lac promoter) and as a control vector pPHU231 were transferred to the $S$. meliloti strains containing the exp-lac $Z$ fusions. $\beta$-Galactosidase activities were determined in the wild-type background, and in the $\Delta \exp G, m u c R$ and $\Delta \exp G-m u c R$ mutants. No differences were observed when the strains lacking the plasmids were compared to strains carrying vector pPHU231. In the wild-type and $\Delta \exp G$ backgrounds, extra copies of the $\exp G$ gene stimulated expression of all five exp-lacZ transcriptional fusions under high phosphate conditions (Fig. 1); the extent differed for each $\exp$ operon. While extra copies of $\exp G$ resulted in $10-25$-fold enhanced $\beta$-galactosidase activities for the

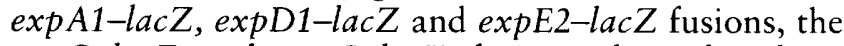

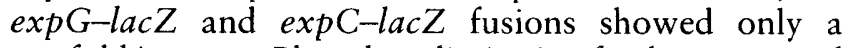
twofold increase. Phosphate limitation further increased 
Table 2. Putative PHO boxes in the promoter regions preceding the $\exp G, \exp A, \exp D$ and $\exp$ operons

The first row shows the E. coli consensus PHO box with two direct repeats separated by an ATrich spacer of two to seven nucleotides (Wanner, 1993, 1996; VanBogelen et al., 1996). The typical consensus sequence for the spacer of E. coli PHO boxes is shown. Weakly conserved nucleotides of the consensus sequence found in promoters shown to be regulated by a phosphorus stimulus

(VanBogelen et al., 1996) are printed in lower case. The second row shows the putative PHO boxes identified by Bardin et al. (1996) preceding the phoC gene. Below, sequences located upstream of the $\exp G, \exp A, \exp D$ and $\exp E$ operons are shown. Nucleotides in these regions which are identical to the consensus PHO box are printed in bold.

\begin{tabular}{|lcccc|}
\hline Gene & Direct repeat & $\begin{array}{c}\text { AT-rich spacer } \\
(\mathbf{2}-\mathbf{7} \mathbf{~ n t})\end{array}$ & Direct repeat & $\begin{array}{c}\text { No. of } \\
\text { nucleotides } \\
\text { to ATG* }\end{array}$ \\
\hline & CTTTCat & AT (A) AT (A) & CTTTCAC & \\
phoC & G & G A T & \\
& CTGTTAC & AGAA & CCTACAC & 84 \\
expG & CTGACAC & TGCG & CTTTCAT & 62 \\
expA1 & GTGTTAT & GAAATTA & CTTCAAG & 38 \\
expD1 & TTTGTCT & GAATATTG & CTTCAAT & 170 \\
expE1 & CTTCTGG & ATAAATCG & ATTTATT & 25 \\
& CTTATCG & CAACAATA & CTGTGAT & 64 \\
& CTCGTCG & CAAATTA & CTTTAAA & 229 \\
\hline
\end{tabular}

* The position of the PHO-box-like sequences is given by the number of nucleotides to the ATG start codon of the relevant gene.

$\beta$-galactosidase levels for all fusions. Under high phosphate conditions it made no difference if extra copies of $\exp G$ were provided by the plasmid pSR1018 or pSR1018-5. However, under low phosphate conditions, the presence of plasmid pSR 1018 carrying the $\exp$ G gene preceded by the native $\exp G$ promoter region caused a slightly higher expression of the expA1-lacZ and

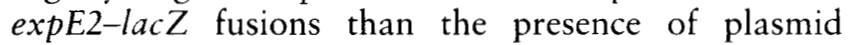
pSR1018-5 containing the $\exp G$ gene preceded by the lac promoter (data not shown).

The $\beta$-galactosidase activities detected in the $\exp G$ deletion mutant $\operatorname{SmSR} \Delta \mathrm{G}$ when extra copies of $\exp G$ were present showed no significant difference in comparison to the wild-type carrying extra copies of $\exp G$. In the $m u c R$ and $\triangle \exp G-m u c R$ backgrounds, extra copies of the $\exp G$ gene did not have any effect on the $\beta$ galactosidase activities mediated by the exp-lacZ fusions (Fig. 1).

\section{PhoB is required for the activation of exp gene transcription under phosphate limitation}

Sequence analysis of promoter regions preceding E. coli genes whose expression is influenced by phosphate resulted in the identification of a consensus sequence for PhoB binding, the so-called PHO box (Makino et al., 1994; VanBogelen et al., 1996). Putative PHO boxes were also identified in the intergenic regions preceding the exp complementation groups (Table 2). Whereas both in front of the $\exp G$ and the $\operatorname{expD1}$ gene one PHO box was found, we identified two putative PHO boxes in front of the $\exp A 1$ and $\exp E 1$ genes. In comparison to the PHO box preceding the $\exp G$ gene the other PHO boxes are only weakly conserved. No PHO-box-like sequence was identified in the intergenic region preceding the $\operatorname{expC}$ gene.

The data showing exp operon induction under phosphate starvation (Fig. 1) and the identification of the sequences with similarities to the $E$. coli $\mathrm{PHO}$ box consensus in the exp promoter regions led to the hypothesis that the response regulator PhoB plays an important role in the phosphate-dependent regulation of exp gene transcription. To investigate this hypothesis, a phoB: : TnV mutation (phoB3) (Bardin \& Finan, 1998) was transferred to $S$. meliloti strains carrying the different exp-lac $Z$ fusions. $\beta$-Galactosidase activities were determined in the exp-lacZ/phoB:: TnV double mutants cultured in MOPS medium containing either $2 \mathrm{mM}$ phosphate or $0.1 \mathrm{mM}$ phosphate (Fig. 2). In the wild-type strain, phosphate limitation resulted in increased $\exp$ gene transcription; however, no effect of phosphate starvation could be detected in the $p h o B$ background. The level of $\beta$-galactosidase activities of all exp-lac $Z$ fusions in the $p h o B$ mutant corresponded to the level of $\beta$-galactosidase activities detected in the wild-type background for the cells grown in MOPS medium with $2 \mathrm{mM}$ phosphate added (Fig. 2).

To analyse if the stimulating effect of $m u c R$ on $\exp$ expression is dependent on $p h o B$, expression of the 


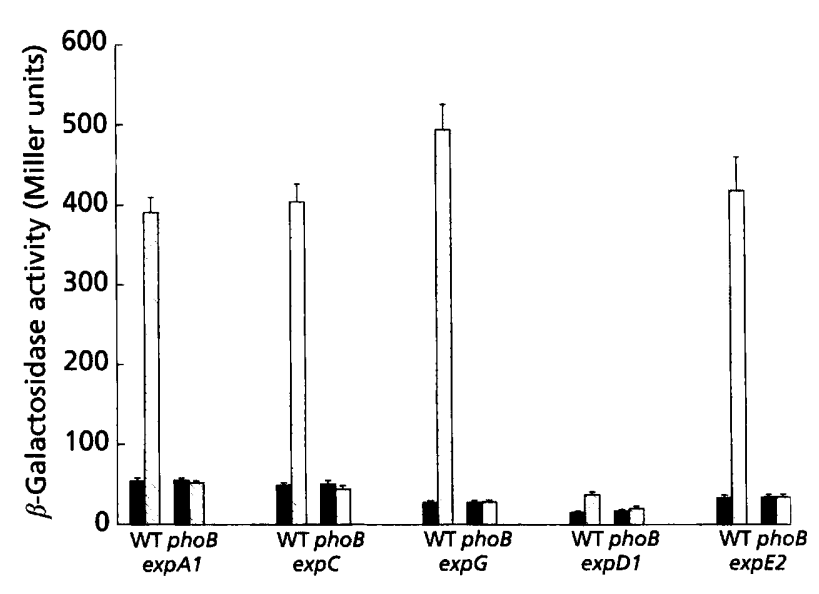

Fig. 2. Effect of a phoB mutation on exp gene expression. Relative $\beta$-galactosidase activities mediated by the

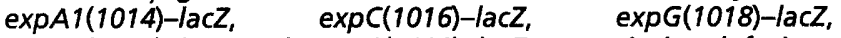
expD1(1020)-lacZ and expE2(1022)-lacZ transcriptional fusions in the wild-type (WT) and phoB::TnV mutant (phoB) strains are shown. The cells were grown in MOPS medium with $2 \mathrm{mM}$ phosphate $(\square)$ or $0.1 \mathrm{mM}$ phosphate $(\mathbb{\nabla})$ added. The $\beta$ galactosidase activity values are means of at least five independent assays. The background $\beta$-galactosidase activity of the wild-type strain Rm2011 was $4 \pm 1$ Miller units. Error bars denote 1 SD.

exp-lacZ fusions was determined in a $m u c R-p h o B$ mutant. In the presence of $2 \mathrm{mM}$ phosphate, exp-lac $Z$ expression was induced at a level comparable to the induction level in a mucR mutant (data not shown). This indicates that $\mathrm{PhoB}$ is not required for the induction of exp expression in a mucR mutant. To analyse if $p h o B$ is required for activation of $\exp$ transcription by $\operatorname{ExpG}$, plasmid pSR1018-5 carrying $\exp G$ preceded by the lac promoter was transferred to the exp-lacZ/phoB mutants. In a $p h o B$ mutant, extra copies of expG induced exp-lac $Z$ expression under high and low phosphate conditions at the same level as additional copies of $\exp G$ induced $\exp -l a c Z$ expression under high phosphate conditions in the wild-type background (data not shown). This indicates that ExpG is able to activate exp transcription independent from PhoB.

\section{DISCUSSION}

Using exp-lacZ transcriptional fusions for each of the five $\exp$ operons, we detected increased exp gene expression in response to phosphate limitation. These results confirm the observation by Zhan et al. (1991), who reported that phosphate limitation induces the production of EPS II. Our data indicate that the phosphate effect on EPS II biosynthesis is at the gene transcription level of all known exp operons. Sequence comparisons of the intergenic regions of the exp operons to promoter regions preceding genes regulated by $\mathrm{PhoB}$ in $E$. coli led to the identification of sequence elements similar to the PHO boxes present in phosphate-regulated promoters of E. coli. Since expression of the exp-lacZ fusions was induced in response to phosphate limitation, it is likely that these putative $\mathrm{PHO}$ boxes located upstream of the $\exp A 1, \exp G, \operatorname{expD1}$ and $\exp E 1$ genes mediate the regulation by a PhoB-like protein in response to phosphate. The phosphate-dependent regulation of the phosphate (PHO) regulon in $E$. coli is controlled by the two-component regulatory system PhoR-PhoB (Wanner, 1993, 1996). Under phosphatelimiting conditions, the sensor PhoR activates the response regulator $\mathrm{PhoB}$ via phosphorylation. $\mathrm{PhoB}$ activates transcription by binding to promoters that are characterized by the PHO box. Recently, a high-affinity phosphate uptake system which is required for an effective symbiotic interaction between $S$. meliloti and alfalfa was identified in S. meliloti (Bardin et al., 1996; Voegele et al., 1997). This transport system is encoded by the phoCDET genes, which are also induced in response to phosphate starvation. Interestingly, analysis of the $p h o C$ promoter led to the identification of two putative PHO boxes. The assumption that the PHO boxes in $S$. meliloti are controlled by a system like PhoR-PhoB was recently supported by the identification of the S. meliloti phoB gene (Al-Niemi et al., 1997; Voegele et al., 1997; Bardin \& Finan, 1998; P. A. McLean and others, GenBank accession no. M96261). Additionally, Bardin \& Finan (1998) showed that $p h o B$ is required for $p h o C D E T$ expression and that $\mathrm{PhoB}$ may also act as a repressor, since the expression of the alternative orf $A$ pit-encoded low-affinity phosphate transport system is negatively regulated by the $p h o B$ gene product. As inferred by the presence of a PHO box preceding genes repressed or induced by phosphate limitation the regulator $\mathrm{PhoB}$ may also have activating or repressing properties in E. coli (VanBogelen et al., 1996). In $S$. meliloti, a phoB mutation abolished induction of the exp-lacZ transcriptional fusions under phosphate-limiting conditions. The facts that (i) exp expression was strongly activated in response to phosphate limitation, (ii) PHO boxes were identified in the exp promoter regions and (iii) $p h o B$ was required for the activation of exp gene transcription under phosphatelimiting conditions strongly suggest that $\mathrm{PhoB}$ functions as an activator of $\exp$ gene transcription.

We previously reported that MucR acts as a transcriptional repressor of the exp genes (Becker et al., 1997). The high levels of $\beta$-galactosidase activities detected in the mucR mutant under high phosphate concentrations ( $2 \mathrm{mM}$ phosphate) were further increased under phosphate limitation $(0 \cdot 1 \mathrm{mM}$ phosphate). This additional increase indicates that MucR may act independently from the phosphate-specific regulatory system, or it influences this system but it is not an essential part thereof. This hypothesis is supported by the finding that $\mathrm{PhoB}$ was dispensable for induction of exp expression in a $m u c R$ mutant.

We recently proposed that ExpG functions as a transcriptional regulator based on similarities to transcriptional regulators of the MarR family (Becker $e$ t al., 1997). This is supported by Astete \& Leigh (1996), who reported that ExpG is essential for the expression of one gene of the $\exp E$ operon under phosphate limitation. In 
the wild-type background additional copies of the $\exp G$ gene resulted in increased exp-lacZ transcription, indicating that ExpG functions as an activator of $\exp$ gene transcription; however, the level of increase differed. Expression levels of $\exp G$ and $\exp C$ were at least five to seven times lower than those of the three other exp genes. Since the effects of extra copies of $\exp G$ on the transcription of $\exp C$ and $\exp G$ were comparable and no PHO-box-like sequence was found upstream of the $\exp C$ coding region, the phosphate- and $\exp G$-dependent regulation of $\operatorname{expC}$ might be mediated by the promoter and the PHO box preceding $\exp G$. The effect of extra copies of $\exp G$ was further increased under phosphate-limiting conditions. This result might be explained by PhoB either acting directly to increase exp gene transcription under phosphate limitation or activating transcription indirectly by first increasing copies of ExpG.

We further showed that a deletion of the $\exp G$ gene had no effect on the phosphate-dependent transcription of

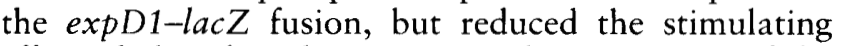
effect of phosphate limitation on the expression of the

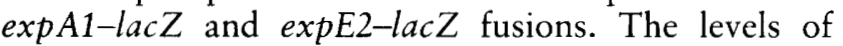
reduction differed for both genes. In particular, the $\operatorname{expE2}-l_{a c} Z$ fusion appeared most influenced by the $\exp G$ deletion. A possible explanation for these results is that under phosphate-limiting conditions the affinity of the response regulator PhoB for the PHO box in the $\exp G$ promoter region is greater than for the less-

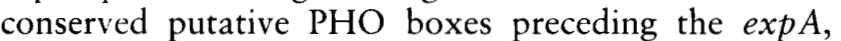
$\operatorname{expD}$ and $\operatorname{expE}$ operons. Relative differences in PhoB affinity for the different $\mathrm{PHO}$ boxes may also explain the function of ExpG, which could act to balance overall $\exp$ expression. As the $\exp G$ deletion did not completely block the stimulation of exp gene expression under phosphate-limiting conditions, we suggest that $\exp G$ is not required for mediating the phosphate signal. Moreover, $p h o B$ was not required for induction of exp expression by ExpG if transcription of $\exp G$ was directed by a constitutive promoter. This indicates that ExpG and PhoB are able to activate exp expression independently from each other.

Three different regulators (MucR, ExpG and PhoB) influencing exp gene expression in $S$. meliloti were identified. Previously, it was shown that MucR has DNA-binding activity. By in vitro experiments binding sites for MucR were identified upstream of the $m u c R$, exo $Y$ and $e x o H$ genes, but not upstream of the five exp operons (Bertram-Drogatz et al., 1997, 1998). This indicates that MucR does not bind directly to the exp promoter regions or that at least one additional factor is necessary to promote binding of MucR. Since PhoB and ExpG were not required for the induction of exp expression in a mucR mutant, it is unlikely that these proteins are necessary to promote binding of MucR. ExpG shares similarities with transcriptional regulators of the MarR family which bind DNA through a helix-turn-helix motif (Cohen et al., 1993; Sulavik et al., 1995), and thus ExpG might regulate exp gene transcription by direct binding to the exp promoter regions. However, binding sites for ExpG remain to be identified.

\section{ACKNOWLEDGEMENTS}

We are grateful to A. Roxlau and T. M. Finan for providing plasmid $\mathrm{pARII} / 1 \mathrm{inv} \Delta \exp G$ and the $p h o B$ mutants, respectively. This work was supported by grant Pu28/17-2 from Deutsche Forschungsgemeinschaft.

\section{REFERENCES}

Al-Niemi, T. S., Summers, M. L., Elkins, J. G., Kahn, M. L. \& McDermott, T.R. (1997). Regulation of the phosphate stress response in Rhizobium meliloti by PhoB. Appl Environ Microbiol 63, 4978-4981.

Astete, S. G. \& Leigh, J. A. (1996). mucS, a gene involved in activation of galactoglucan (EPS II) synthesis gene expression in Rbizobium meliloti. Mol Plant-Microbe Interact 9, 395-400.

Bardin, S. \& Finan, T. M. (1998). Regulation of phosphate assimilation in Rhizobium (Sinorhizobium) meliloti. Genetics 148, 1689-1700.

Bardin, S., Dan, S., Osteras, M. \& Finan, T. M. (1996). A phosphate transport system is required for symbiotic nitrogen fixation by Rhizobium meliloti. J Bacteriol 178, 4540-4547.

Battisti, L., Lara, J. C. \& Leigh, J. A. (1992). Specific oligosaccharide form of the Rhizobium meliloti exopolysaccharide promotes nodule invasion in alfalfa. Proc Natl Acad Sci USA 89, 5625-5629.

Becker, A., Kleickmann, A., Arnold, W. \& Pühler, A. (1993a). Analysis of the Rhizobium meliloti exoH/exoK/exoL fragment: ExoK shows homology to excreted endo- $\beta-1,3-1,4-$ glucanases and ExoH resembles membrane proteins. Mol Gen Genet 238, 145-154.

Becker, A., Kleickmann, A., Arnold, W., Keller, M. \& Pühler, A. (1993b). Identification and analysis of the Rhizobium meliloti exoAMONP genes involved in exopolysaccharide biosynthesis and mapping of promoters located on the exoHKLAMONP fragment. Mol Gen Genet 241, 367-379.

Becker, A., Kleickmann, A., Küster, H., Keller, M., Arnold, W. \& Pühler, A. (1993C). Analysis of the Rhizobium meliloti genes exoU, exoV, exoW, exoT and exol involved in exopolysaccharide biosynthesis and nodule invasion: exoU and exoW probably encode glycosyltransferases. Mol Plant-Microbe Interact 6, 735-744.

Becker, A., Niehaus, K. \& Pühler, A. (1995a). Low molecular weight succinoglycan is predominantly produced by Rhizobium meliloti strains carrying a mutated ExoP protein characterized by a periplasmic $\mathrm{N}$-terminal and a missing C-terminal domain. Mol Microbiol 16, 191-203.

Becker, A., Küster, H., Niehaus, K. \& Pühler, A. (1995b). Extension of the Rhizobium meliloti succinoglycan biosynthesis gene cluster: identification of the exs $A$ gene encoding an $\mathrm{ABC}$ transporter protein, and the exsB gene which probably codes for a regulator of succinoglycan biosynthesis. Mol Gen Genet 249, $487-497$.

Becker, A., Schmidt, M., Jäger, W. \& Pühler, A. (1995c). New gentamicin resistance and lacZ promoter probe cassettes suitable for insertion mutagenesis and generation of transcriptional fusions. Gene 162, 37-39.

Becker, A., Rüberg, S., Küster, H., Roxlau, A., Keller, M., Ivashina, T., Cheng, H.-P., Walker, G. C. \& Puhler, A. (1997). The 32-kilobase exp gene cluster of Rhizobium meliloti directing the biosynthesis 
of galactoglucan: genetic organization and properties of the encoded gene products. J Bacteriol 179, 1375-1384.

Beringer, J. E. (1974). $\mathrm{R}$ factor transfer in Rhizobium leguminosarum. J Gen Microbiol 84, 188-198.

Bertram-Drogatz, P. A., Rüberg, S., Becker, A. \& Pühler, A. (1997). The regulatory protein $M u c R$ binds to a short DNA region located upstream of the $m u c R$ coding region in Rbizobium meliloti. Mol Gen Genet 254, 529-538.

Bertram-Drogatz, P. A., Quester, I., Becker, A. \& Pühler, A. (1998). The Sinorhizobium meliloti MucR protein, which is essential for the production of high-molecular-weight succinoglycan exopolysaccharide, binds to short DNA regions upstream of exoH and exoY. Mol Gen Genet 257, 433-441.

Buendia, A. M., Enenkel, B., Köplin, R., Niehaus, K., Arnold, W. \& Pühler, A. (1991). The Rbizobium meliloti exoZ/exoB fragment of megaplasmid 2: ExoB functions as a UDP-glucose-4-epimerase and ExoZ shows homology to NodX of Rhizobium leguminosarum biovar viceae strain TOM. Mol Microbiol 5, 1519-1530.

Bullock, W. C., Fernandez, J. M. \& Short, J. M. (1987). XL1-Blue: a high efficiency plasmid transforming recA Escherichia coli strain with beta-galactosidase selection. BioTechniques $\mathbf{5}$, 376-379.

Casse, F., Boucher, C., Hulliot, J. S., Michel, M. \& Dénarié, J. (1979). Identification and characterization of large plasmids in Rhizobium meliloti using agarose gel electrophoresis. J Bacteriol 113, 229-242.

Cohen, S. P., Hachler, H. \& Levy, S. B. (1993). Genetic and functional analysis of the multiple antibiotic resistance (mar) locus in Escherichia coli. J Bacteriol 175, 1484-1492.

Dehoux, P. \& Cossart, P. (1995). Homologies between salmolysin and some bacterial regulatory proteins. Mol Microbiol 15, 591-592.

Finan, T. M., Hartwieg, E., LeMieux, K., Bergman, K., Walker, G. C. \& Signer, E. R. (1984). General transduction in Rhizobium meliloti. J Bacteriol 159, 120-124.

Glazebrook, J. \& Walker, G. C. (1989). A novel exopolysaccharide can function in place of the Calcofluor-binding exopolysaccharide in nodulation of alfalfa by Rhizobium meliloti. Cell 56, 661-672.

Glucksmann, M. A., Reuber, T. L. \& Walker, G. C. (1993a). Family of glycosyl transferases needed for the synthesis of succinoglycan by Rhizobium meliloti. J Bacteriol 175, 7033-7044.

Glucksmann, M. A., Reuber, T. L. \& Walker, G. C. (1993b). Genes needed for the modification, polymerization, export, and processing of succinoglycan by Rhizobium meliloti: a model for succinoglycan biosynthesis. J Bacteriol 175, 7045-7055.

González, J. E., Reuhs, B. L. \& Walker, G. C. (1996). Low molecular weight EPS II of Rhizobium meliloti allows nodule invasion in Medicago sativa. Proc Natl Acad Sci USA 93, 8636-8641.

Hübner, P., Willison, J. C., Vignais, P. M. \& Bickle, T. A. (1991). Expression of regulatory nif genes in Rhodobacter capsulatus. J Bacteriol 173, 2993-2999.

Keller, M., Roxlau, A., Weng, W. M., Schmidt, M., Quandt, J., Niehaus, K., Jording, D., Arnold, W. \& Pühler, A. (1995). Molecular analysis of the Rhizobium meliloti mucR gene regulating the biosynthesis of the exopolysaccharides succinoglycan and galactoglucan. Mol Plant-Microbe Interact 8, 267-277.

Kessler, C. (1992). Nonradioactive Labeling and Detection of Biomolecules. Berlin: Springer.

Leigh, J. A. \& Walker, G. C. (1994). Exopolysaccharides of Rhizobium: synthesis, regulation and symbiotic function. Trends Genet 10, 63-67.
Leigh, J. A., Signer, E. R. \& Walker, G. C. (1985). Exopolysaccharide-deficient mutants of Rbizobium meliloti that form ineffective nodules. Proc Natl Acad Sci USA 82, 6231-6234.

Makino, K., Amemura, M., Kim, S., Nakata, A. \& Shinagawa, H. (1994). Mechanism of transcriptional activation of the phosphate regulon in Escherichia coli. In Phosphate in Microorganisms, pp. 5-12. Edited by A. Torriani-Gorini, E. Yagil \& S. Silver. Washington, DC: American Society for Microbiology.

Marklund, B.-I., Tennent, J. M., Garcia, E., Hamers, A., Baga, M., Lindberg, F., Gaastra, W. \& Normark, S. (1992). Horizontal gene transfer of the Escherichia coli pap and prs pili operons as a mechanism for the development of tissue-specific adhesive properties. Mol Microbiol 6, 2225-2242.

Masepohl, B., Klipp, W. \& Püler, A. (1988). Genetic characterization and sequence analysis of the duplicated nif $A /$ nif $B$ gene region of Rhodobacter capsulatus. Mol Gen Genet 212, 27-37.

Meade, H. M., Long, S. R., Ruvkun, G. B., Brown, S. E. \& Ausubel, F. M. (1982). Physical and genetic characterization of symbiotic and auxotrophic mutants of Rhizobium meliloti induced by transposon Tn5 mutagenesis. J Bacteriol 149, 114-122.

Miller, J. H. (1972). Experiments in Molecular Genetics. Cold Spring Harbor, NY: Cold Spring Harbor Laboratory.

Muller, P., Keller, M., Weng, W. M., Quandt, J., Arnold, W. \& Puhler, A. (1993). Genetic analysis of the Rhizobium meliloti exoYFQ operon: ExoY is homologous to sugar transferases and ExoQ represents a transmembrane protein. Mol Plant-Microbe Interact 6, 55-65.

Reinhold, B. B., Chan, S. Y., Reuber, T. L., Marra, A., Walker, G. C. \& Reinhold, V. N. (1994). Detailed structural characterization of succinoglycan, the major exopolysaccharide of Rhizobium meliloti Rm1021. J Bacteriol 176, 1997-2002.

Reuber, T. L. \& Walker, G. C. (1993). Biosynthesis of succinoglycan, a symbiotically important exopolysaccharide of Rhizobium meliloti. Cell 74, 269-280.

Sambrook, J., Fritsch, E. F. \& Maniatis, T. (1989). Molecular Cloning: a Laboratory Manual, 2nd edn. Cold Spring Harbor, NY : Cold Spring Harbor Laboratory.

Schäfer, A., Tauch, A., Jäger, W., Kalinowski, J., Thierbach, G. \& Pühler, A. (1994). Small mobilizable multi-purpose cloning vectors derived from the Escherichia coli plasmids pK18 and pK19: selection of defined deletions in the chromosome of Corynebacterium glutamicum. Gene 145, 69-73.

Simon, R. (1984). High frequency mobilization of gram-negative bacterial replicons by the in vitro constructed Tn5-Mob transposon. Mol Gen Genet 196, 413-420.

Simon, R., Priefer, U. \& Püler, A. (1983). A broad host range mobilization system for in vivo genetic engineering: transposon mutagenesis in gram-negative bacteria. Bio/Technology 1, 784-791.

Sulavik, M. C., Gambino, L. F. \& Miller, P. F. (1995). The MarR repressor of the multiple antibiotic resistance (mar) operon in Escherichia coli: prototypic member of a family of bacterial regulatory proteins involved in sensing phenolic compounds. Mol Med 1, 436-446.

VanBogelen, R. A., Olson, E. R., Wanner, B. L. \& Neidhardt, F. C. (1996). Global analysis of proteins synthesized during phosphorus restriction in Escherichia coli. J Bacteriol 178, 4344-4366.

Voegele, R. T., Bardin, S. \& Finan, T. M. (1997). Characterization of the Rbizobium (Sinorbizobium) meliloti high- and low-affinity phosphate uptake systems. J Bacteriol 179, 7226-7232.

Wanner, B. L. (1993). Gene regulation by phosphate in enteric bacteria. J Cell Biochem 51, 47-54. 
Wanner, B. L. (1996). Phosphorus assimilation and control of the phosphate regulon. In Escherichia coli and Salmonella: Cellular and Molecular Biology, 2nd edn, pp. 1357-1381. Edited by F. C. Neidhardt and others. Washington, DC: American Society for Microbiology.

Zhan, H., Levery, S. B., Lee, C. C. \& Leigh, J. A. (1989). A second exopolysaccharide of Rhizobium meliloti strain SU47 that can function in root nodule invasion. Proc Natl Acad Sci USA 86, 3055-3059.

Zhan, H., Lee, C. C. \& Leigh, J. A. (1991). Induction of the second exopolysaccharide (EPSb) in Rhizobium meliloti strain SU47 by low phosphate concentrations. J Bacteriol 173, 7391-7394.

Zimmermann, J., Voss, H., Schwager, C., Stegemann, J., Erfle, H., Stucky, K., Kristensen, T. \& Ansorge, W. (1990). A simplified method protocol for fast plasmid DNA sequencing. Nucleic Acids Res 18, 1067.

Received 17 August 1998; revised 2 November 1998; accepted 19 November 1998. 